\title{
Reading of "De Rerum Natura” in the Light of Modern Physics
}

\author{
Gualtiero Pisent \\ Istituto Nazionale di Fisica Nucleare, Sezione di Padova, Padova, Italy \\ Email: Pisent@pd.infn.it
}

Received April 28 $8^{\text {th }}$, 2012; revised May 30 ${ }^{\text {th }}$, 2012; accepted June $10^{\text {th }}, 2012$

\begin{abstract}
An analysis of the Lucretius atomism is given, that makes particular reference to the naturalistic arguments and contents of the poem. A possible comparison with the atomism of nowadays, based on quite new, both theoretical (quantum mechanics) and experimental (particle accelerators) grounds, must be treated with much care. But it seems possible and interesting to compare at least the world outlined by Lucretius, with the new world, derived by familiarity with modern theories of matter. This is the point I have tried to stress.
\end{abstract}

Keywords: Science; Nature; Atomism; Vacuum; Swerve

\section{Introduction}

Although Lucretius theories are not fully original (being derived in particular from Epicurus), the poem "De rerum natura" is so wide, self consistent and miraculously integral in the form arrived up to our days, that it is worthwhile to be studied and analyzed as a whole.

The literature about the poem is very wide, and only some titles are given here for easy reference (Costa, 1984; Kenney, 1971; Smith, 1992)

The interests that promoted these comments are mainly philosophical, poetical, philological and historical, while the aim of this work is to start directly from the original text, and try to stress the naturalistic approach of Lucretius, in comparison with the current ideas about the structure of matter.

It is worthwhile to mention in particular an article recently published in Physics Today (Gruijv, 2012), that starts the "Insights on the classical atoms” from Democritus and Lucretius, reporting among others the following considerations:

"As fertile as it was, the atomic hypothesis suffered from a major deficiency: its authors happened to be atheists. Subsequent generations were reluctant to accept the atom, and the concept remained buried in religious animosity for nearly two millennia.”

Also with reference to this feature of the question, namely the physics and the meta physics of Lucretius, I hope that some light should be shed by the present analysis.

\section{From Lucretius to Avogadro}

The following analysis will be carried out through comments on selected quotations of the text (Caro, 1989).

The numeration refers to the Latin edition of the poem.

The translation from Latin to English is more "ad sensum" than "word for word".

The first book, on "the Matter", prepares the audience to accept the invisible atoms as real components of the world.

"Perhaps you'll not believe to my words, because these primitive matter components cannot be detected by eyes. But I'll give you clear examples of things that are certainly in the world, and cannot be seen. The first example is given by the gust of wind, which lashes the sea, strikes the ships and dragsthe clouds [I-265].”

We shall see later on, the reasons that suggest the atomistic idea, but first of all we must be aware that the assumption, although not trivial, is nevertheless realistic.

"The clothes, left on the seaside, become wet. Then, under the sunshine, they get dry. But how is water able to penetrate the cloth, and how to leave it, nobody can see [I-305].”

The arguments make reference to the water and the steam.

The second argument involves explicitly the evaporation phenomenon, which is a convincing example of something that we feel as real but we don't see by eyes.

It is interesting to note (and it is not casual I guess) that when (after eighteen centuries) the atomistic philosophical assumption becomes a physical theory, this happens because Amedeo Avogadro (1776-1856) was investigating about the chemistry of the gaseous state.

Among the three states of matter condensation (solid liquid and gas), the latter one happens to have the far simplest structure, and therefore it was, in the history of science, the natural tool to inquire how the microcosm works.

Avogadro doesn't see air, like Lucretius didn't, but Avogadro can work on the interpretation of Boyle's and Gay Lussac's experiments.

He owns a technology able to operate on gases by pressure and temperature variations, and to detect some of their peculiar properties, without seeing them.

So for the first time in history, (thanks to the discovery of the famous Avogadro's number), mankind learned that the molecule of a gas, is about $10 \exp \{-8\} \mathrm{cm}$ wide.

Lucretius had no idea about the atom's dimensions but certainly he had no reason to suspect that they were so small.

Note that, up to this point, we used the word "atom" in the sense of Lucretius, namely to designate a microscopic constituent of matter, not better identified. Now, with reference to Avogadro, we must say "molecules" because, as it is well known, the chemistry of the gaseous state introduced atoms and molecules, and discovered that only the molecules of the so called noble gases, are mono atomic.

From now on we'll continue to speak of atoms with reference 
to Lucretius, but whenever the modern theory of matter will be referred to, we'll introduce the words molecule, atom, nucleus, electron and so on, in the well defined, modern meaning.

Preliminarily to the introduction of his atoms, Lucretius had two problems to solve. The first one was the compactness of matter, which seemed to be an obstacle to the atom's mobility, and the second one was the gravity effect, which seemed to hinder the atomic collisions.

Much space was given by Lucretius to these questions, which are solved very naturally by modern physics (these points do not represent serious objections nowadays).

Let us start with the famous "vacuum" problem.

"All things in nature are not at all compact; there is inside bodies the vacuum .... otherwise, no kind of motion of things should be possible [I-329]”.

The following statement tries to justify the assumption of hollow spaces inside matter, that we never can see by eyes, nor experience directly in some way.

"It is common experience that some bodies are heavier than others of equal volume .... The heavier body clearly contains less quantity of vacuum inside [I-358]".

The line of reasoning is very subtle: the atomic structure itself suggests the idea of a matter constant in density. If this is the case, heavier bodies may exist near lighter ones, only because they contain a lower amount of empty spaces inside.

It should be interesting but difficult to comment this statement in the light of the modern knowledges, because the structure of the microcosm happened to be much more complicated than expected. Three levels were discovered in the micro world, namely the molecular and the atomic levels (both dominated by the electromagnetic interaction, and populated by objects as wide as $10 \exp \{-8\} \mathrm{cm}$ ), and the nuclear level (dominated by the strong interaction and populated by objects as wide as 10 $\exp \{-13\} \mathrm{cm})$. In particular the nuclear density is extremely high, namely it is equivalent to one aircraft carrier per cubic millimeter, and this means that inside each atom, large hollow spaces are left to the flight of the electrons. This kind of vacuum was clearly beyond any possible imagination at the time of Lucretius.

Then, after a long introduction, the author lastly presents his main player, namely the atom:

"Bodies are composed by bodies. But we must find something not capable of further decomposition. If an object like this exists, it must be solid and timeless [I-624]”.

It follows a very important sentence about the true meaning and the behavior of this object inside the matter:

"Movements of atoms do not follow primeval projects or shrewd indications of some mind in the universe. They have tried and experienced from eternity to now, a huge number of paths, collisions, deflections, up to find their final state corresponding to the present structure of the world [I-1021]".

Here we have first something that may be interpreted as a declaration of atheism in a modern sense: the final state of order reached by the world, is not the effect of Somebody who has thought and realized a project.

Then it follows the description of what really happened.

The latter statement shows prophetic analogies with the big bang theory. To explain what I mean, I report a sentence taken from the famous book of Weinberg on "The first three minutes" (Weinberg, 1977):

"First frame: the temperature of the Universe is of 100 billions of Kelvin degrees ... The universe is full of matter and radiation, and each particle undertakes very fast collisions with the others ... we have electrons and positrons, photons, neutrinos and anti neutrinos".

The words of Weinberg seem to furnish us the scientific details, about something, that was well described under wide general lines, by Lucretius.

But there is much more about the mechanisms of evolution, in the quoted phrases.

Sometimes, during a natural development of a system subject to purely stochastic rules, certain configurations are realized, whose occurrence seems to be a priori extremely improbable. But what seems a miracle, is simply explained by the long time that evolution left to the universe, to try a huge number of attempts.

This concept, very important in physics, has been widely discussed by Jacques Monod, with reference to biology (Monod, 1971).

The second book of the poem, on "the Atoms", entered into the details of the dynamics of atoms; here much attention is given to the gravity effects, which were considered by Lucretius as an important and unavoidable background in the whole cosmic phenomenology.

"Bodies in space can not move spontaneously from down to up ... And this is not contradicted by the blaze of the burning objects ... Also golden cereals and trees seem to be projected toward the sky, but heavy bodies cannot avoid falling down ... Wooden planks are projected up from water, but fall down in vacuum [II-184]”.

The line of reasoning is very clear: the nature of simple bodies is to fall down. Something may, on the contrary, be pushed up, but only as a consequence of complicated mechanisms. Nothing in nature is simpler than atoms, "ergo" atoms fall down anyway.

It is interesting to analyze the counter examples listed by Lucretius. The first mechanism able to push something up, is the floating of the wood in water: Archimedes (287-212 BC) is not explicitly mentioned, but his famous principle was well known at the time of Lucretius. The mechanism of the blaze is along the same line, although a little more complicated (air is heated and climbs, dragging up the blaze itself). But the growing up of cereals and trees, belongs to quite another phenomenology: we deal here with living objects (at least at a simple botanical level), able to distinguish between up and down by virtue of complicated sensory receptors.

This is a first symptom of a very peculiar feature of Lucretius philosophy: his atomism first introduced with reference to inert matter, is then naturally extended to the living bodies and to the mind. Modern science is much more careful when dealing with this kind of extension.

At this stage the very important concept of "clinamen" (swerve) were introduced into the theory by Lucretius:

"While atoms are driven by their weight in straight vertical falls through the vacuum, they happen to be casually deflected from their trajectory. In absence of this swerve, they should fall, regularly and eternally, like rain drops; no atomic collision, and consequently no process of generation of the world, should have been possible [II-216]”.

In other words, the presence of the gravitation on the earth, was perceived by Lucretius as a severe obstacle to his theory. His atoms were seen as small material objects, that fall like rain drops, on vertical parallel trajectories. If this was the picture, it was clearly hard to imagine how collisions between atoms 
could take place, and this is the reason why he introduced the swerve.

But nowadays, we know exactly the mass and dimensions of the gas molecules in the air. They are much smaller and lighter than Lucretius could imagine, and the consequence is that gravity is totally ineffective on their dynamics.

The mathematical character of modern physics allows us to demonstrate the statement by a calculation.

Let us consider, for example, 1 mole of helium gas, enclosed in a cubic box, at normal conditions of temperature and pressure. On the basis of the classical theory of ideal gases (describing the gas molecules as point-like non-interacting particles), it is easy to calculate the average kinetic energy of one molecule, namely:

$$
\mathrm{E}=(3 / 2) \mathrm{KT}=56 \times 10 \exp (-22) \text { joule }
$$

(where $\mathrm{T}$ is the absolute temperature and

$\mathrm{K}=1.38 \times 10 \exp (-23)$ [joule/Kelvin degree], the Boltzmann constant). On the same footing, we are able to evaluate the difference in gravitational energy between a molecule on the top and a molecule on the bottom of the box, namely:

$$
\mathrm{U}=\mathrm{mgh}=18 \times 10 \exp (-27) \text { joule }
$$

where $\mathrm{m}=6.64 \times 10 \exp (-27) \mathrm{kg}$, is the mass of the helium molecule, $g=9.81(\mathrm{~m} / \mathrm{sec} \exp \{2\})$, is the gravity acceleration and $\mathrm{h}=0.282 \mathrm{~m}$, is the height of the cube containing the mole of gas.

We can finally calculate the ratio $U / E=1 / 300,000$, which gives the order of magnitude of the contribution of the gravity effects on the particle dynamics. The very small value of the ratio, clearly indicates that the phenomenon is totally unaffected by gravity.

For the details on the calculations, one can see any treatise of classical thermodynamics, (Piragino, 1983). Our numerical results refer of course to a particular example, and depend on the chosen physical conditions; but we are interested only in the order of magnitude of the results, which doesn't change very much.

If one tries to calculate the molecular dynamics within the liquid or solid (instead of gaseous) state, the job should become much more difficult, because the strong inter molecular forces should be taken into account.

But in the latter case, just the internal bounds typical of the condensed states, should make weaker and weaker the contribution of the gravitational field to the whole dynamics.

Therefore we can say that the above demonstration has wide limits of generality.

The conclusion is that particles fly free like butterflies, describing perfect straight lines (whose directions are randomly distributed in space) between particle-particle or particle-wall collisions. The random motion is perfectly suitable to generate collisions and molecular reactions, without the help of any "clinamen".

It is worthwhile at this point, to precise better our viewpoint of modern scientists, about the world outlined by Lucretius.

First of all Lucretius had perfectly in mind the picture of a chemical reaction within the gaseous state, as it is described by modern science, but this idea was hardly disturbed by the image of the rain drops, and the rain drops were the natural phenomenon, closer to what he thought would happen at the invisible, microscopic level. His error was derived from an overestimation of the atomic mass, while modern physics shows that his picture was realistic in spite of gravity, and therefore without necessity of introducing the swerve.

\section{From the Matter to the Soul}

But this is the point that draws on a new (substantially unjustified but very fascinating) line, the whole philosophical system of Lucretius.

Because, once the new actor (namely "clinamen”) was introduced, it was immediately called by the author, to play a very fundamental role in the whole universe dynamics, as it is explained by the following sentence:

"If all atoms movements were internal to a continuous stiff chain, the new ones deriving with inexorable destiny from the previous ones, which may be the origin of the freedom, granted to anybody is breathing on the earth? Where does our will come from, which is driven by our pleasure's search and drives our actions, not subjected to fortune's laws? ... Everything derives from this light swerve, which affects the atomic movements in spaces and times that nobody controls [II-251]”.

As noted before, a fundamental contradiction inside the old atomism, comes from the continuity of the chain of logic that goes from inert matter, to living bodies and to human mind, without any gap.

Lucretius was very aware that somewhere in the chain, it was necessary to introduce some kind of discontinuity, able to explain the gap between blind motion of matter and intelligent human life, and used to solve this problem, the brilliant idea of the swerve.

Once this serious problem seemed to be satisfactory solved, he went on (in the third book of the poem, on "the Death"), to the discussion of the atomic structure of the soul, in the same framework as he previously discussed the atomic structure of inert matter:

"What is it possible to say about the soul's nature? ... No dynamics in the world is more speedy than the process that takes place when the mind formulates a project, and acts on the body to start the relative practical operations... The subject which is responsible for a so rapid and complicated dynamics, must be composed by spherically shaped and extremely small and light elements [III-177]”.

There follows in the poem, a very interesting comparison among water (a liquid with a low viscosity), honey (which clearly exhibits a much more dense and viscous flow), and the things of the ghost. If all of these are rings of a certain logical chain, all composed by atoms, the atoms of different rings can not be of the same type. They must exhibit, along the chain, properties of increasing lightness and mobility, as far as one proceeds from matter to ghost.

Lucretius's atomism is naively global and all-inclusive, and leads naturally to a very rough and worldwide kind of materialism:

"Since the soul is part of the body, in the sense that it occupies a precise position within the body, like the eyes, the ears and the other sensor organs, ... we conclude that the soul can not exist without the body and the man, who behaves like a vessel or a container [III-548]".

In other words the soul dies with the body, and this docks the poem to higher level considerations "de consolatione philosophiae”.

"Death is nothing for us and doesn't belong to us, if deadly is the soul ... When we'll cease existing, after the divorce be- 
tween soul and body whose junction makes our being, be sure that nothing will be able to reach our senses, even if ground and sea or sea and sky will mix up [III-830]”.

I mentioned already that "atom's movements do not follow projects or indications of some mind in the universe". The protection offered by the old gods is therefore rejected, but it is immediately substituted by the encouraging idea that nothing bad (or better nothing at all) can happen to us after death.

Nowadays atomism is based on stiff grounds, both theoreticcal (mathematics) and experimental (instruments for acceleration and detection of particles); but these grounds are stiff just because their field of validity is rigorously auto-limited. The new scientific language is built up around the part of the world that evolves in such a way that the laws of increasing total entropy are fulfilled. On the other side, the biological world, whose development is locally anti-entropic, and especially the world of the mind, must be treated with much care, on a quite separate footing.

My conclusion is that, in spite of the obvious difficulties of reading Lucretius (fully justified by twenty centuries of distance) the poetical descriptions of both the brutal matter and the divine soul, conserve fully the original fascination and wisdom, with an important remark. Whilst the physics of the poem looks (at least somewhere) miraculously prophetic with respect to the representation of the world that we have now clear in our mind, much more care is needed in dealing with the associated meta physics, whose fundamental points seem to be neither convincing in the old poem, nor satisfactorily solved nowadays (certainly not as satisfactorily as the physics seems to be).

\section{REFERENCES}

Costa, C. D. N. (1984). Introduction, Lucretius: De Rerum Natura V. Oxford: Oxford University Press.

Kenney, E. J. (1971), “Introduction”, Lucretius: De Rerum Natura. Cambridge: Cambridge University Press.

Smith, M. (1992). “Introduction”, De Rerum Natura. Cambridge: Loeb Classical Library.

Gruijc, P., \& Simonovic, N. (2012). Insight from the classical atom. Physics Today, 65, 41.

Caro, T. L. (1989). La Natura (Latin text and Italian translation). Milano: Garzanti Editore.

Weinberg, S. (1977). I primi tre minuti. Milan: Mondadori.

Monod, J. (1971). Il caso e la necessit'a. Milan: Mondadori.

Piragino, G., \& Pisent, G. (1983). Fisica generale e sperimentale (Vol. 1). Padova: Piccin Editore. 